\title{
The effect of lattice damage and annealing conditions on the hyperfine structure of ion implanted bismuth donors in silicon
}

\author{
T. Peach, Juerong. Li, S. Chick, B. N. Murdin, and S. K. Clowes \\ Department of Physics, University of Surrey, Guildford, Surrey, GU2 7XH, UK \\ K. P. Homewood \\ Department of Electrical and Electronic Engineering, \\ University of Surrey, Guildford, Surrey, GU2 7XH, UK \\ School of Materials Science $\& 3$ Engineering, \\ Hubei University, Wuhan 430062, PR China and \\ Materials Research Institute and School of Physics and Astronomy, \\ Queen Mary University of London, \\ Mile End Road, E1 4NS London, UK
}

M. A. Lourenco

Department of Electrical and Electronic Engineering,

University of Surrey, Guildford, Surrey, GU2 7XH, UK

M. Hughes

Department of Physics, University of Surrey,

Guildford, Surrey, GU2 7XH, UK and

Joule Physics Laboratory, School of Computing Science and Engineering, University of Salford, Salford M5 4WT, UK

K. Saeedi and N. Stavrias

Radboud University, Institute for Molecules and Materials,

FELIX Laboratory, Toernooiveld $7 c$,

6525 ED Nijmegen, The Netherlands

(Dated: September 17, 2018) 


\begin{abstract}
This study reports on high energy bismuth ion implantation into silicon with a particular emphasis on the effect that annealing conditions have on the observed hyperfine structure of the $\mathrm{Si}: \mathrm{Bi}$ donor state. We observe a suppression of donor bound exciton, $D^{0} X$, photoluminescence in implanted samples which have been annealed at $700^{\circ} \mathrm{C}$ relating to the presence of a dense layer of lattice defects that is formed during the implantation process. 10K Hall measurements show that this implant damage manifests itself at low temperatures as an abundance of p-type charge carriers, the density of which is observed to have a strong dependence on annealing temperature. Using resonant $D^{0} X$ photoconductivity we are able to identify the presence of hyperfine structure in samples annealed at a minimum temperature of $800^{\circ} \mathrm{C}$, however higher temperatures are required to eliminate effects of implantation strain.
\end{abstract}




\section{INTRODUCTION}

Group $\mathrm{V}$ donor impurity centres in silicon indicate vast potential for integration into quantum technologies due to their long measured electron and nuclear spin coherence times and existing compatibility within the electronics industry. Moreover, phosphorus impurities can be engineered in such a way that allows for the possibility of atomically precise positioning with an accuracy of \pm 1 lattice site which has been utilised in the fabrication of such devices as single atom transistors [1] and single atom quantum gates [2]. Bismuth donors in silicon have gained significant interest as a quantum technology platform due to their extremely strong hyperfine interaction and large nuclear spin which manifests as a 20-dimensional nuclear spin Hilbert space and a $30.51 \mu \mathrm{eV}$ ground state hyperfine splitting that is resolvable even in the absence of a magnetic field [3]. These properties allow for a strong coupling of the electron and nuclear spins, making it possible to transfer quantum information quickly between spins [4] in a quantum computer memory, or to other impurities [5] in a quantum gate. They also provide so-called clock transitions where the electron spin resonance is protected from magnetic field noise from the environment [6] and routes to optical pumping for atomic clock state preparation [7]. However, unlike phosphorus, there is as yet no mechanism for deterministically incorporating bismuth donors within a silicon lattice. While ion implantation is a non-deterministic method of depositing group V impurities in silicon, there is a large degree of control over the depth and spatial density of implanted donors through careful selection of the implantation energy and beam fluence respectively. Ion implantation has been used to produce phosphorus quantum devices [8, 9] and single ion implantation has also been demonstrated to display the high degree of control associated with this fabrication procedure $[10,11]$. Moreover small numbers of bismuth atoms have been implanted for spin resonance experiments [12] and the capability to achieve very high resolution implants of single bismuth is currently being developed [13, 14] although a greater understanding of the post implant treatment for substrates is required. This is partly due to the current lack of understanding about the post implantation activation process.

Typically studies of the electrical activation yield, EAY, of bismuth donors in silicon have been focused on high fluence implants $\left(1 \times 10^{14} \mathrm{~cm}^{-2}\right)$ using low energies where it has been shown that an effective method is to use solid phase epitaxial regrowth, SPER. During SPER samples are heated to relatively low temperatures $\left(600^{\circ} \mathrm{C}\right)$ for minute timescales, this allows 
for a full recrystallisation of the amorphous layer that is created during implantation and an incorporation of ions onto lattice sites with yields of up to $90 \%$ and minimal diffusion [15]. However for devices with quantum technology applications it is desirable for donor densities to be much lower in order to preserve long spin coherence times. Further studies, using lower bismuth implantation doses $\left(1 \times 10^{12} \mathrm{~cm}^{-2}\right)$, have shown that by annealing substrates at varying temperatures and durations bismuth activation yields are achieved ranging from $24 \%$ to $67 \%$ with the maximum corresponding to a temperature of $900{ }^{\circ} \mathrm{C}$ and duration of 15 minutes [16]. In the same study, using electron spin resonance, ESR, measurements, the $T_{2}$ time of implanted bismuth donors has been shown to be $0.7 \mathrm{~ms}$ (at $10 \mathrm{~K}$ ) which allows for the possibility of incorporating bismuth in conjunction with phosphorus without significantly degrading the coherence time of the system. Furthermore it has been demonstrated that samples co-implanted with both phosphorus and bismuth impurities do not have significantly different substrate band gap energies when compared to equivalent single donor systems [17]. This therefore may enable optically gated qubit schemes as differing donor species would not be able to interact through ground states due to their differing binding energies $(70.98 \mathrm{meV}$ and $45.59 \mathrm{meV}$ in the example of bismuth and phosphorus impurities respectively [18, 19]). However entanglement would become possible via spatially overlapping excited level wavefunctions that are accessible optically.

To fabricate a large scale bismuth donor layer that is well separated from the substrate surface the implantation energies used in this study are far higher than those implemented in previous studies. High energy ions can cause secondary defect sites and even cascades of vacancies when they collide with target atoms. This may result in localised amorphicity within the implant region, however often up to $98 \%$ of damage is immediately repaired due to a process known as spontaneous annealing [20]. The rate of this depends on numerous factors including the temperature of the substrate during the implantation and the structural integrity of the surrounding lattice area. Previous studies observe that high energy implantation procedures do not cause long range defects and furthermore it has been shown, using a $0.76 \mathrm{MeV} \mathrm{As}^{+}$implantation, that a full crystal recovery can be achieved through furnace annealing [21].

In this work we implement room temperature high energy bismuth ion implantation to fabricate co-doped $\mathrm{Si}: \mathrm{Bi}, \mathrm{P}$ samples using various annealing temperatures to repair damage to the silicon crystal and encourage bismuth incorporation. We have used these samples 
TABLE I. Implantation energies and fluences used in this study.

\begin{tabular}{ccc}
\hline \hline $\begin{array}{c}\text { Implant } \\
\#\end{array}$ & $\begin{array}{c}\text { Energy } \\
(\mathrm{keV})\end{array}$ & $\begin{array}{c}\text { Fluence } \\
\left(10^{12} \mathrm{~cm}^{-2}\right)\end{array}$ \\
\hline 1 & 2000 & 6.12 \\
2 & 1400 & 2.40 \\
3 & 1000 & 2.40 \\
4 & 700 & 1.38 \\
5 & 500 & 1.08 \\
\hline \hline
\end{tabular}

to explore the optimum anneal temperatures to maximise the EAY of implanted bismuth donors via spreading resistance profiling, SRP, measurements [22]. In addition we have verified the co-existence of both phosphorus and bismuth impurities using donor bound exciton, $D^{0} X$, photoluminescence and explored the extent of implantation lattice damage using low temperature Hall measurements. Finally, to probe into the presence of the the zero field ground state hyperfine spin splitting, resonant $D^{0} X$ photoconductivity measurements are also implemented. Consequently we evaluate whether ion implanted bismuth donors in silicon provide a suitable alternative to bulk doped Si:Bi for applications within quantum technologies.

Donor bound excitons are formed when electrons are excited from the valence band to form excitons, $X$, which then become bound to neutral donor sites, $D^{0}$. This complex therefore contains four constituent elements: a positively charged bismuth donor; two electrons; and a hole which together have a resonant formation energy of $1.1469 \mathrm{eV}$ [23] and two possible recombination channels. In bulk doped Si:Bi the dominant mechanism for $D^{0} X$ recombination is the Auger process whereby the exciton recombines and provides enough energy to the donor to ionise into the conduction band where it can be measured electrically using photoconductivity. [24]. The second, much weaker, process is donor bound exciton photoluminescence where the recombination energy is emitted and measured optically [25]. Both of these recombination mechanisms are utilised in in this study to report on the observation of implanted bismuth $D^{0} X$ signals and hence confirm the presence of electrically active bismuth impurities and evaluate their quality of incorporation with a specific emphasis on the ground state hyperfine spin doublet. 


\section{EXPERIMENTAL DETAIL}

Silicon wafers used in this study were $300 \mu \mathrm{m}$ thick, $\langle 111\rangle$ oriented float-zone silicon with a resistivity of 7-8 $\Omega \mathrm{cm}$ due to bulk doped phosphorus impurities with a volume density of $6 \times 10^{14} \mathrm{~cm}^{-3}$. Samples were fabricated using a chain of 5 room temperature bismuth implantation steps as indicated in table I. Following bismuth implantation, substrates were annealed for $300 \mathrm{~s}$ at temperatures of either $700{ }^{\circ} \mathrm{C} 800{ }^{\circ} \mathrm{C}$ or $900{ }^{\circ} \mathrm{C}$. This implant scheme was chosen in order to replicate the properties of bulk Si:Bi in an implanted substrate. Beam fluences were chosen such that resulting donor distributions are high density but below the metal-insulator transition. It was necessary to use a chain of 5 individual implant steps in order to ensure that the absolute number of donor states formed is high enough as to be measurable macroscopically.

During SRP samples were beveled at an angle of $0.005^{\circ}$ whereby the two probe resistivity across the sample was measured using a $5 \mathrm{mV}$ voltage source at $4 \mu \mathrm{m}$ iterative steps down the beveled edge to determine the active donor concentration as a function of depth at room temperature. Luminescence measurements were undertaken using above bandgap photoexcitation provided by a $200 \mathrm{~mW}, 532 \mathrm{~nm}$ laser incident on samples mounted within a liquid helium cooled continuous flow cryostat held at a temperature of $10 \mathrm{~K}$. The emitted luminescence signal was analysed using a 1m SPEX spectrometer using a slit width of $1 \mathrm{~mm}$ and measured using a germanium detector. Hall measurements in this study were performed using magnetic fields of $\pm 2 \mathrm{~T}$ at temperatures ranging from $10 \mathrm{~K}$ to $24 \mathrm{~K}$. Samples were fashioned into a square geometry and silver contacts were deposited at each corner onto the surface of the substrate to allow for resistivity values to be measured using both standard Hall and Van der Pauw orientations [26]. Contacts were deposited onto the surface of the silicon substrate to specifically address the transport properties of charge carriers within the implanted bismuth layer region. Photoconductivity measurements were used to resonantly probe into implanted $\mathrm{Si} B \mathrm{Bi} D^{0} X$ states using a $1 \mu \mathrm{m}$, amplified, tunable external cavity diode laser source with an output power of $80 \mathrm{~mW}$. Samples were held in a a LHe cooled bath cryostat at a temperature of $10 \mathrm{~K}$ and electrical signal was measured using an AC biased lock in technique. Samples were contacted non-intrusively using a capacitor plate geometry to avoid inducing additional strain on the hyperfine structure [27]. 


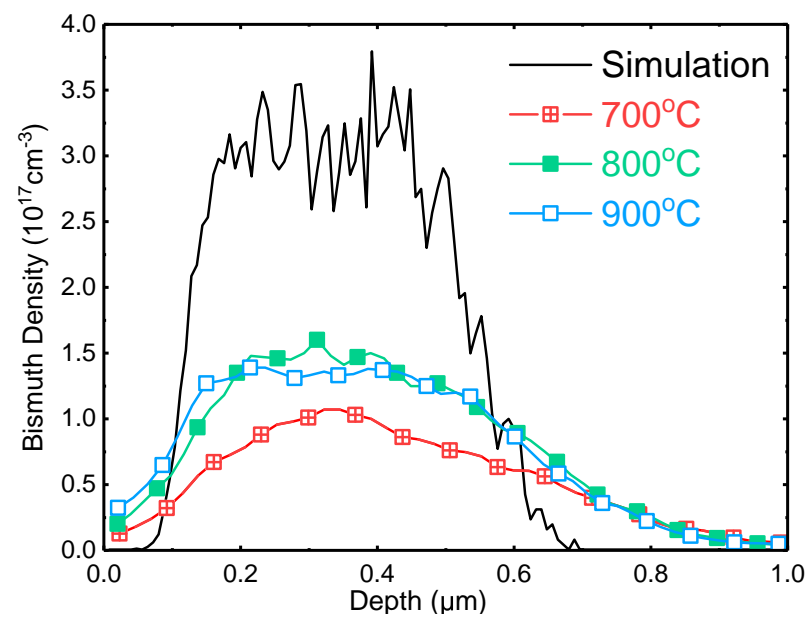

FIG. 1. Active donor density profiles of implanted bismuth samples. The solid black line indicates a monte carlo simulation of the cumulative bismuth donor density displayed in table I. SRP measurements of equivalent samples annealed at temperatures of $900{ }^{\circ} \mathrm{C}, 800{ }^{\circ} \mathrm{C}$ and $700{ }^{\circ} \mathrm{C}$ are also indicated.

\section{RESULTS AND DISCUSSION}

In order to determine the active bismuth concentration as a fraction of the implant dose SRP measurements were utilised and are displayed in figure 1. A SRIM [28] monte carlo simulation is also depicted (solid black line) as a reference indicator for a 'perfect' implant profile assuming $100 \%$ EAY and zero diffusion. This simulation predicts that implants should correspond to a peak bismuth density of $3 \times 10^{17} \mathrm{~cm}^{-3}$ however it is clear that in our measured data there is a reduction of approximately $50 \%$ in the maximum active donor concentration. We also observab that diffusion of the implanted ions has also occurred as a significant amount of active centres are measured to be at depths greater than $600 \mathrm{~nm}$. By calculating the integrated area of each profile displayed in figure 1, the EAY of the corresponding implants have been evaluated and are displayed in table II. Our results for EAY as a function of annealing temperatures are consistent with other studies using similar implant doses [16]. We observe that the yield increases as a function of anneal temperature and appears to plateau at an approximate value of $60 \%$ using a temperature of $900{ }^{\circ} \mathrm{C}$ for 300 s. The limit we observe in EAY corresponds to the donor concentration approaching the solid solubility limit of bismuth donors in silicon $\left(2.3 \times 10^{17} \mathrm{~cm}^{-3}[29]\right)$. For each of the implanted samples displayed in figure $1, D^{0} X$ photoluminescence measurements have 
TABLE II. Electrical activation yield of implanted bismuth donors in silicon with various annealing temperatures for $300 \mathrm{~s}$.

\begin{tabular}{cc}
\hline \hline Anneal Temp & EAY \\
$\left({ }^{\circ} \mathrm{C}\right)$ & $(\%)$ \\
\hline 900 & $59 \pm 12$ \\
800 & $53 \pm 11$ \\
700 & $41 \pm 8$ \\
\hline \hline
\end{tabular}

been implemented and corresponding spectra are displayed in figure 2. An un-implanted control spectrum is also indicated. Peaks corresponding to luminescence from the transverse phonon replicas of both phosphorus and bismuth donor bound exciton centres are shown, Si:P BE(TO) and Si:Bi BE(TO) respectively. We do not present the equivalent zero phonon peaks as they are in close proximity to the transverse acoustic phonon assisted bandgap transition in silicon whereas both the BE(TO) lines are observable unobstructed. The Si:P $\mathrm{BE}(\mathrm{TO})$ is clearly present in all four spectra as every substrate used in this study contains bulk doped phosphorus impurities with a constant volume density. Therefore this signal has been used to normalise all spectra for the purpose of comparing the equivalent spectra. Figure 2 indicates that the luminescence centre of the $\mathrm{Si}: \mathrm{Bi} D^{0} X$ has a strong dependence on the annealing temperature such that for the sample annealed at $700{ }^{\circ} \mathrm{C}$ no strength is visible whereas the peak emerges for increasing temperatures. To explore the correlation between substrate annealing temperature and $D^{0} X$ luminescence intensity, Hall measurements have been implemented over a magnetic field range of $\pm 2 \mathrm{~T}$ at temperatures spanning $10 \mathrm{~K}$ to $24 \mathrm{~K}$. We determine that measured values for the Hall resistivity, $\rho_{x y}$, indicate the presence of multiple types of charge carriers however two carriers appear to dominate the conduction in this temperature range. Therefore the two carrier Hall resistivity model [30] is implemented such that,

$$
\rho_{x y}=\frac{\sigma_{x y}}{\sigma_{x y}^{2}+\sigma_{x x}^{2}}
$$

where $\sigma_{x y}$ and $\sigma_{x x}$ are Hall conductivity values perpendicular and parallel to the direction of applied current respectively. $\sigma_{x y}$ and $\sigma_{x x}$ are expressed in equations 2 and 3 ,

$$
\sigma_{x y}=\frac{n e \mu_{e}^{2} B}{1+\mu_{e}^{2} B^{2}}-\frac{p e \mu_{h}^{2} B}{1+\mu_{h}^{2} B^{2}}
$$




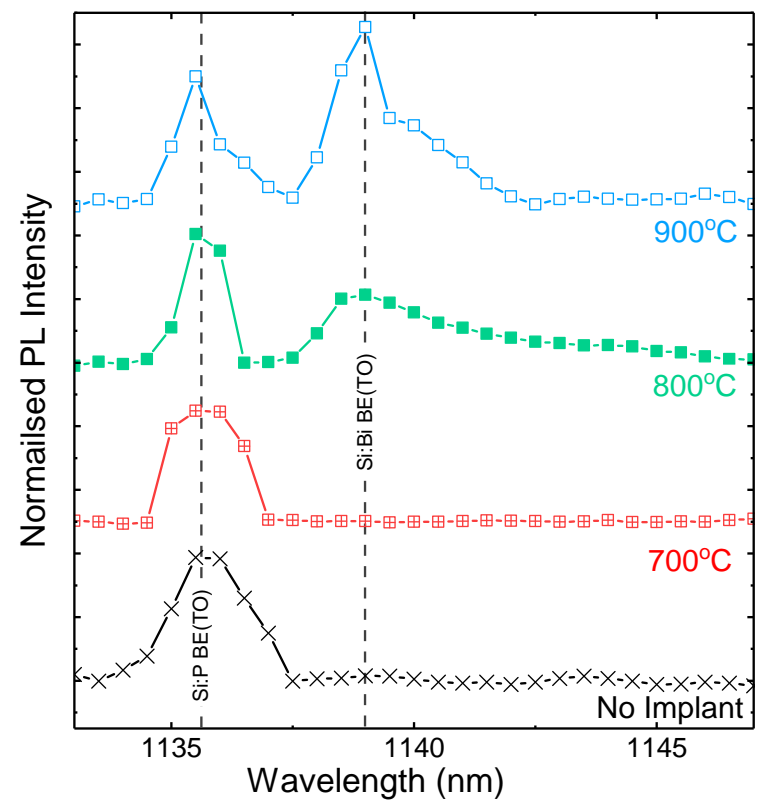

FIG. 2. Above bandgap photoluminescence spectra of implanted bismuth samples with annealing temperatures as indicated for $300 \mathrm{~s}$. Indicated peaks correspond to the transverse optical phonon replicas of both phosphorus and bismuth impurity donor bound exciton luminescence centres, Si:P $\mathrm{BE}(\mathrm{TO})$ and $\mathrm{Si}: \mathrm{Bi} \mathrm{BE}(\mathrm{TO})$ respectively.

$$
\sigma_{x x}=\frac{n e \mu_{e}}{1+\mu_{e}^{2} B^{2}}+\frac{p e \mu_{h}}{1+\mu_{h}^{2} B^{2}}
$$

where $n$ and $\mu_{e}$ are n-type carrier sheet densities and mobilities respectively and $p$ and $\mu_{h}$ are equivalent p-type parameters. To distinguish between contributions of the two charge types, equations 1-3 were used to fit measured $\rho_{x y}$ values. In an effort to reduce the dimension of the fitting parameter space, $n$ was calculated using $p, \mu_{e}$ and $\mu_{h}$ in conjunction with the zero field Van der Pauw resistivity $\left(\rho_{x x}=1 / \sigma_{x x}(B=0)\right)$ which was measured at each temperature iteration. 


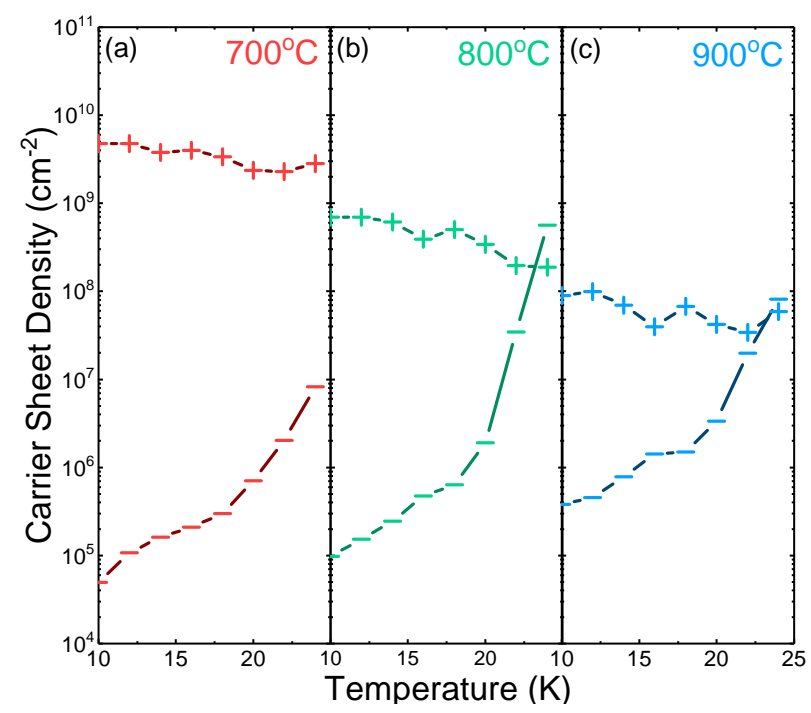

FIG. 3. (a), (b) \& (c) Low temperature charge carrier sheet concentrations for samples annealed at $700{ }^{\circ} \mathrm{C}, 800{ }^{\circ} \mathrm{C}$ and $900{ }^{\circ} \mathrm{C}$ respectively. Markers displayed as " + " indicate the density of holes whereas " - " correspond to electrons.

Figure 3 presents that, at low temperatures, the Hall resistivity for each sample contains contributions from the conduction of both electrons and holes. By observation it is clear that the measured charge carrier densities far exceed that which would be allowed at $10 \mathrm{~K}$ by the law of mass action [31]. We must therefore infer that the origin of these differing carriers are spatially separated into two distinct population regions between which there is limited charge recombination. Moreover it is apparent that annealing the implanted substrate at higher temperatures corresponds to a decrease in p-type charge carrier density which suggests that the mechanism of which holes are being created is directly related to damage incurred by the lattice during ion implantation. However it is also notable that even for samples annealed at the highest temperature a significant proportion of p-type carriers remain active. It has been shown using Rutherford backscattering spectrometry that during ion implantation a thin, heavily damaged layer is generated at the surface of the substrate with an amorphisation rate that is proportional to the nuclear energy deposition at the interface [32]. We therefore reason that the origin of measured p-type charge transport is a high density surface layer of lattice defects which act as acceptor centres that are caused by the implantation. Conversely, the measured electron signal can be attributed to the bulk doped phosphorus donors of which a very small fraction be ionised at $10 \mathrm{~K}$. It is also 
TABLE III. 10K carrier densities and mobilities extracted using equations 2 and 3 for n-type and p-type charges.

\begin{tabular}{ccccc}
\hline \hline Annealing & $n$ & $\mu_{e}$ & $p$ & $\mu_{h}$ \\
Temperature & $\times 10^{5}$ & $\times 10^{4}$ & $\times 10^{7}$ & $\times 10^{2}$ \\
$\left({ }^{\circ} \mathrm{C}\right)$ & $\left(\mathrm{cm}^{-2}\right)$ & $\left(\mathrm{cm}^{2} \mathrm{~V}^{-2} \mathrm{~s}^{-1}\right)$ & $\left(\mathrm{cm}^{-2}\right)$ & $\left(\mathrm{cm}^{2} \mathrm{~V}^{-2} \mathrm{~s}^{-1}\right)$ \\
\hline 700 & $0.50 \pm 0.02$ & $10.7 \pm 0.4$ & $474.1 \pm 0.1$ & $0.5484 \pm 0.0002$ \\
800 & $0.98 \pm 0.02$ & $9.9 \pm 0.2$ & $69.82 \pm 0.06$ & $2.905 \pm 0.002$ \\
900 & $3.5 \pm 0.1$ & $9.0 \pm 0.3$ & $8.42 \pm 0.02$ & $16.59 \pm 0.04$ \\
\hline \hline
\end{tabular}

apparent that the concentration of n-type carriers in all three samples measured increases with temperature whereas the density of p-type charges remains relatively constant. This can be credited to the increasing population of ionised phosphorus donors as temperature rises.

Table III displays the determined values of both n-type and p-type carrier concentrations at a temperature of $10 \mathrm{~K}$ along with corresponding mobility values for each charge type. The sample annealed at $700{ }^{\circ} \mathrm{C}$ displays, at $10 \mathrm{~K}$, a sheet concentration of p-type charge carriers that is approximately 5 orders of magnitude higher than the density of corresponding n-type carriers. Furthermore, in the presence of a more dense population of p-type lattice defects a reduction of n-type charge carriers is also observed and is attributed to an increase in charge recombination at the interface between the layers.

It is clear that there is a correlation between the population of observed p-type defects and the strength of Si:Bi PL emission. This is such that the $700{ }^{\circ} \mathrm{C}$ substrate records the highest population of holes but there is a complete suppression in luminescence. We can therefore suggest that the presence of implantation defects is causing a suppression of this center by providing parallel non-radiative recombination channels. Furthermore, given the EAY values displayed in table II, the intensity of spectra from the luminescence of implanted bismuth impurities annealed at $800{ }^{\circ} \mathrm{C}$ is lower than would be expected when compared to the corresponding $900{ }^{\circ} \mathrm{C}$ signal. This indicates that, although luminescence is clearly observed for both samples, it is likely that each centre is also being suppressed to some extent by the presence of lattice defects. Additionally shown in table III is that the mobility of p-type carriers also depends strongly on annealing temperature. It is clear that for samples 
annealed at $700{ }^{\circ} \mathrm{C}, \mu_{h}$ is greatly reduced when compared to the corresponding $900{ }^{\circ} \mathrm{C}$ annealed value. This can be accounted for considering that as the density of p-type lattice defects decreases, the number of hole scattering events also decreases and corresponding mobility values increase.

In order to more fully evaluate the candidacy of ion implanted bismuth donors in silicon as spin qubits, it is necessary to explore the effect that the implantation procedure has on the nature of the zero-field hyperfine spin splitting. Furthermore it is essential to determine the optimum annealing conditions to promote bismuth incorporation such that donor states are unaffected by implant strain. Samples used in this measurement have depth profiles identical to those discussed previously in this study however use a slightly reduced peak active bismuth donor concentration of $6 \times 10^{16} \mathrm{~cm}^{-3}$ to eliminate any potential concentration broadening effects. Figure 4 displays the measured photoconductivity signal as a function of pump laser energy.

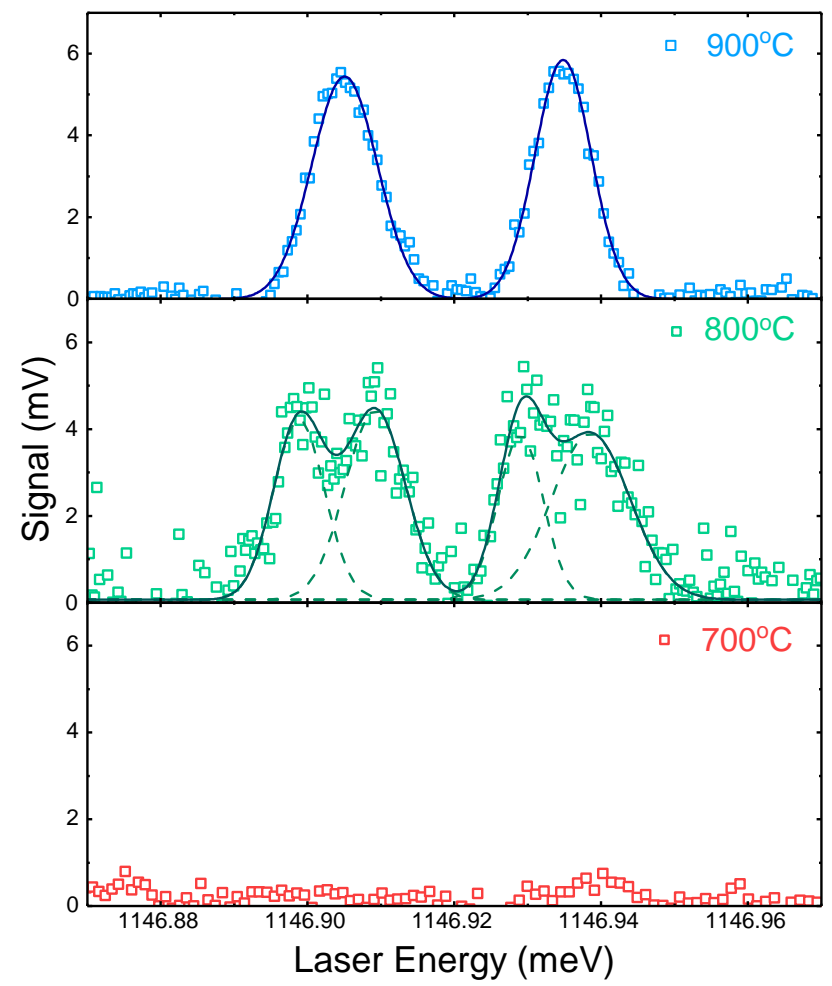

FIG. 4. Resonant $D^{0} X$ photoconductivity spectra of implanted Si:Bi samples annealed for $300 \mathrm{~s}$ with temperatures as indicated.

There is a clear correlation between the quality of observed $D^{0} X$ spectra and substrate 
annealing temperature. This is such that samples annealed at $700{ }^{\circ} \mathrm{C}$ show no signal corresponding to bismuth impurities whatsoever. This observation is consistent with the absence of PL spectra observed in figure 2 for the corresponding $700{ }^{\circ} \mathrm{C}$ annealed sample, and can be related to the abundance of implant related defect states presented in figure 3. As annealing temperature increases the bismuth donor spin states begin to emerge. However the spectra taken from samples annealed at $800{ }^{\circ} \mathrm{C}$ differs significantly from that which would

be expected in bulk Si:Bi. We measure additional structure within the $D^{0} X$ spectrum such that further splitting within the hyperfine states are observed. While the exact mechanism creating this strain is not very well understood, it is clear that there is a correlation between the post implantation annealing conditions and the 'quality' of $D^{0} X$ spectra generated. We therefore infer that the channel through which these additional undesirable features are formed is related to strain generated during the implantation procedure and is linked to the presence of acceptor defect states presented in figure 3. Finally, using an annealing temperature of $900{ }^{\circ} \mathrm{C}$ we are able to clearly resolve the zero-field hyperfine doublet that is associated with bismuth donors in silicon. By fitting each peak using a Gaussian distribution we measure an energy splitting of $(29.84 \pm 0.05) \mu \mathrm{eV}$ between the two states with corresponding linewidths of $(8.93 \pm 0.09) \mu \mathrm{eV}$ and $(7.68 \pm 0.08) \mu \mathrm{eV}$ FWHM for the low and high energy peaks respectively. These measured values are in very good agreement with equivalent parameters extracted from bulk doped Si:Bi substrates; $30.51 \mu \mathrm{eV}$ and $7.9 \mu \mathrm{eV}$ for spacing and linewidths respectively [33]. This suggests that, while implantation defects remain present in this substrate, the applied annealing regime is sufficient to both encourage bismuth incorporation and reduce implantation strain such that the groundstate hyperfine doublet is resolvable and comparable to equivalent bulk doped $\mathrm{Si} B \mathrm{Bi}$ samples.

\section{CONCLUSION}

To conclude we have studied the characteristics of implanted co-doped Si:Bi,P substrates using various bismuth fluences and annealing temperatures. Using spreading resistance profiling measurements we have determined that the electrical activation yield of implanted donors increases with annealing temperature. Using both above bandgap photoluminescence and resonant $D^{0} X$ photoconductivity measurements we observe a strong correlation between annealing temperature and the quality of bismuth donor incorporation which is 
more complex than solely considering the EAY. Using low temperature Hall measurements we are able to relate this to the presence of lattice defects caused by the ion implantation procedure which manifest as an abundance of p-type charge carriers. It is found that annealing conditions of $900{ }^{\circ} \mathrm{C}$ for $300 \mathrm{~s}$ was sufficient to incorporate implanted bismuth donors such that their ground state hyperfine doublet is resolvable, unperturbed and highly comparable with equivalent bulk Si:Bi samples. This therefore gives confidence that ion implantation is a viable method of fabricating bismuth donors in silicon for integration into quantum technologies.

We gratefully acknowledge the financial support from the UK Engineering and Physical Sciences Research Council [COMPASSS/ADDRFSS, Grant No.EP/M009564/1]. B.N.M. is grateful for a Royal Society Wolfson Research Merit Award. T.P. is grateful for his SEPnet PhD studentship.

[1] M. Fuechsle, J. a. Miwa, S. Mahapatra, H. Ryu, S. Lee, O. Warschkow, L. C. L. Hollenberg, G. Klimeck, and M. Y. Simmons, Nature Nanotechnology 7, 242 (2012).

[2] Y. Wang, A. Tankasala, L. C. L. Hollenberg, G. Klimeck, M. Y. Simmons, and R. Rahman, npj Quantum Information 2, 16008 (2016).

[3] G. Feher, Phys. Rev. 114, 1219 (1959).

[4] G. W. Morley, M. Warner, a. M. Stoneham, P. T. Greenland, J. Van Tol, C. W. M. Kay, and G. Aeppli, Nature Materials 9, 725 (2010).

[5] A. M. Stoneham, A. J. Fisher, and P. T. Greenland, J. Phys.: Condens. Matter 15, 447 (2003).

[6] G. Wolfowicz, A. M. Tyryshkin, R. E. George, H. Riemann, N. V. Abrosimov, P. Becker, H.-J. Pohl, M. L. W. Thewalt, S. A. Lyon, and J. J. L. Morton, Nature Nanotechnology 8, 881 (2013), arXiv:1301.6567.

[7] K. Saeedi, M. Szech, P. Dluhy, J. Z. Salvail, K. J. Morse, H. Riemann, N. V. Abrosimov, N. Nötzel, K. L. Litvinenko, B. N. Murdin, and M. L. W. Thewalt, Scientific Reports 5, 10493 (2015). 
[8] E. Prati, K. Kumagai, M. Hori, and T. Shinada, Scientific Reports 105, 246804 (2016).

[9] E. Prati, M. Hori, F. Guagliardo, G. Ferrari, and T. Shinada, Nature Nanotechnology 7, 443 (2012).

[10] T. Shinada, S. Okamoto, T. Kobayashi, and I. Ohdomari, Nature 437, 1128 (2005).

[11] D. N. Jamieson, C. Yang, T. Hopf, S. M. Hearne, C. I. Pakes, S. Prawer, M. Mitic, E. Gauja, S. E. Andresen, A. S. Dzurak, and R. G. Clark, Applied Physics Letters 86, 202101 (2005).

[12] A. Bienfait, J. J. Pla, Y. Kubo, M. Stern, X. Zhou, C. C. Lo, C. D. Weis, T. Schenkel, M. L. W. Thewalt, D. Vion, D. Esteve, B. Julsgaard, K. Mølmer, J. J. L. Morton, and P. Bertet, Nature Nanotechnology 11, 253 (2016).

[13] EPSRC Grant EP/N015215/1 Quantum technology capital: Multi-species single-ion implantation: http://gow.epsrc.ac.uk.

[14] M. Ilg, C. D. Weis, J. Schwartz, A. Persaud, Q. Ji, C. C. Lo, J. Bokor, A. Hegyi, E. Guliyev, I. W. Rangelow, and T. Schenkel, Journal of Vacuum Science \& Technology B 4, 4 (2017).

[15] J. P. De Souza and P. F. P. Fichtner, Journal of Applied Physics 74, 119 (1993).

[16] C. D. Weis, C. C. Lo, V. Lang, A. M. Tyryshkin, R. E. George, K. M. Yu, J. Bokor, S. A. Lyon, J. J. L. Morton, and T. Schenkel, Applied Physics Letters 100, 172104 (2012).

[17] C. M. Araújo, J. Souza de Almeida, I. Pepe, A. F. da Silva, B. E. Sernelius, J. P. de Souza, and H. Boudinov, Phys. Rev. B 62, 882 (2000).

[18] N. R. Butler, P. Fisher, and A. K. Ramdas, Phys. Rev. B 12, 3200 (1975).

[19] A. K. Ramdas and S. Rodriguez, Rep. Prog. Phys. 44, 1297 (1981).

[20] J. F. Ziegler, Nuclear Instruments and Methods in Physics Research 6, 270 (1985).

[21] E. F. Krimmel, H. Oppolzer, H. Runge, and W. Wondrak, Phys. Stat. Sol. 66, 565 (1981).

[22] A. Othonos, C. Christofides, and J. Boussey-Said, Journal of Applied Physics 75, 8032 (1994).

[23] P. J. Dean, W. F. Flggd, and G. Kaminsky, 163, 721 (1967).

[24] D. F. Nelson, J. D. Cuthbert, P. J. Dean, and D. G. Thomas, Phys. Rev. Lett. 17, 1262 (1966).

[25] J. R. Haynes, Physical Review Letters 4, 361 (1960).

[26] L. van der Pauw, Philips Research Reports 13, 1 (1958).

[27] K. Saeedi, M. Steger, M. L. W. Thewalt, N. Abrosimov, H. Riemann, P. Becker, and H. J. Pohl, AIP Conference Proceedings 1566, 552 (2013).

[28] J. F. Ziegler, "Interactions of ions with matter: http://www.srim.org,". 
[29] F. A. Trumbore, Bell System Technical Journal 39, 205 (1960).

[30] W. A. Beck, J. R. Anderson, and A. Beck, Journal of Applied Physics 62, 541 (1987).

[31] N. W. Ashcroft and D. N. Mermin, Solid State Physics (1976).

[32] T. Lohner, M. Fried, N. Q. Khánh, P. PetriK, H. Wormeester, and M. A. El-Sherbiny, Nuclear Instruments and Methods in Physics Research B 147, 90 (1999).

[33] T. Sekiguchi, M. Steger, K. Saeedi, M. L. W. Thewalt, H. Riemann, N. V. Abrosimov, and N. Nötzel, Physical Review Letters 104 (2010).

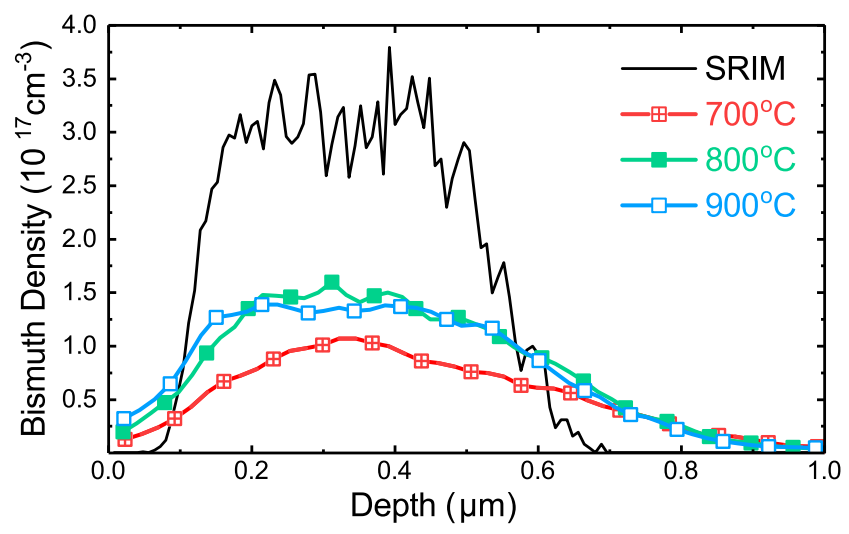

FIG. 5. 


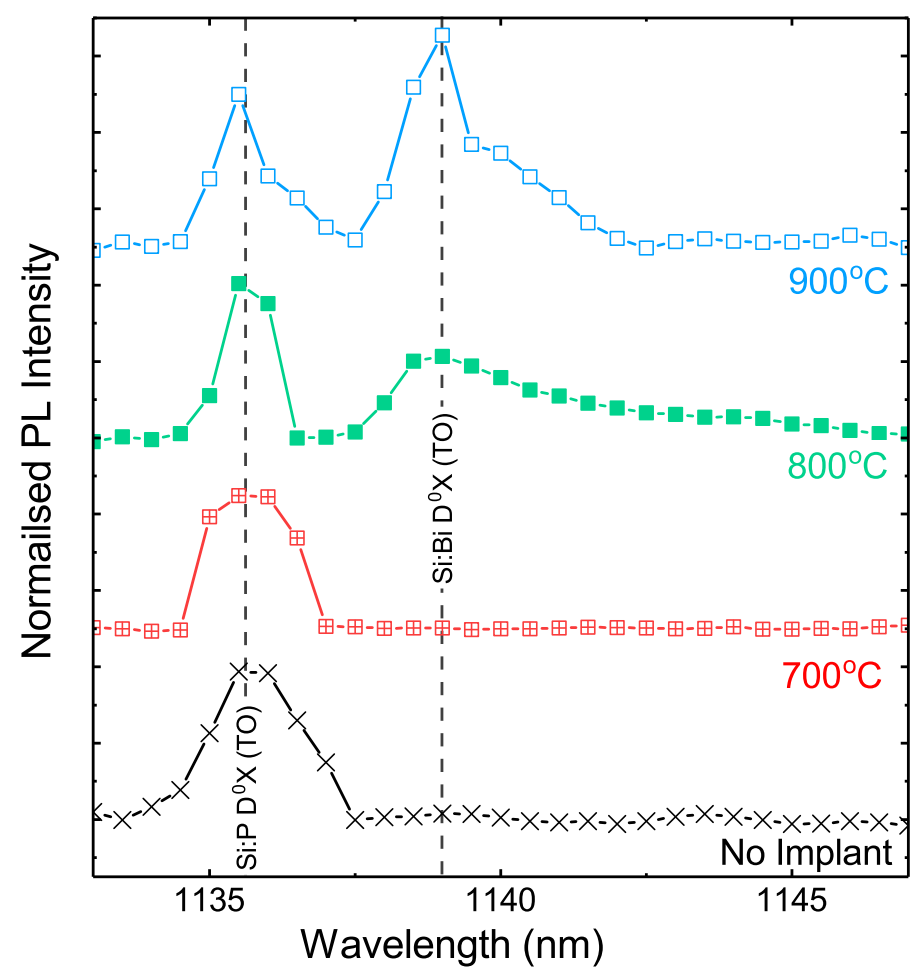

FIG. 6.

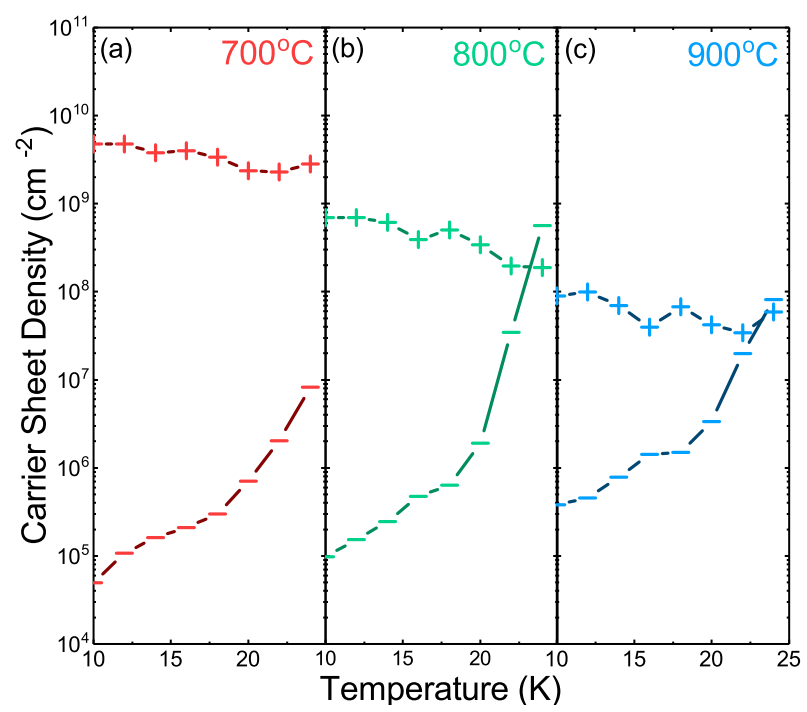

FIG. 7. 


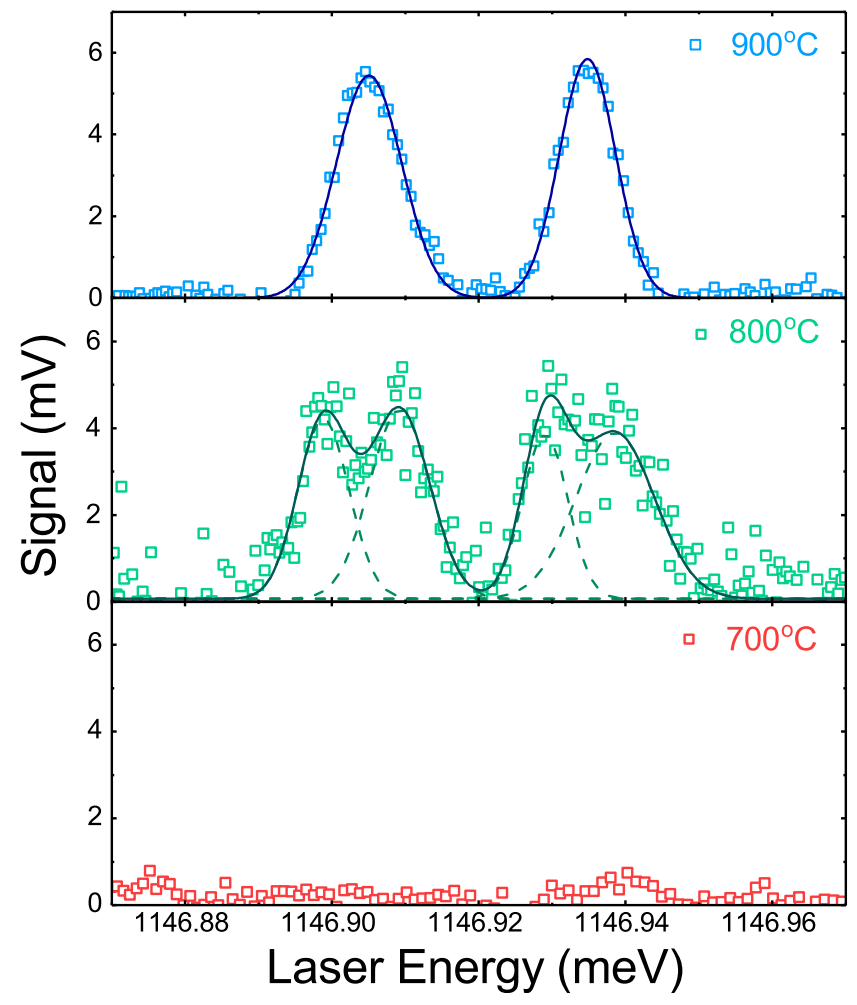

FIG. 8.

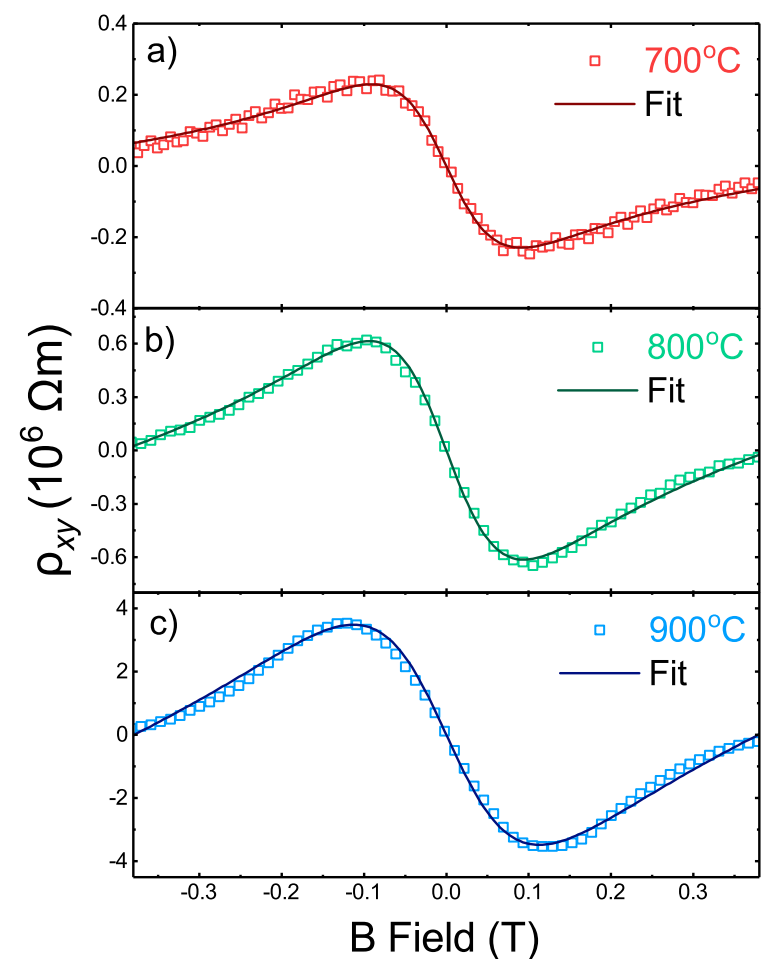

FIG. 9. 


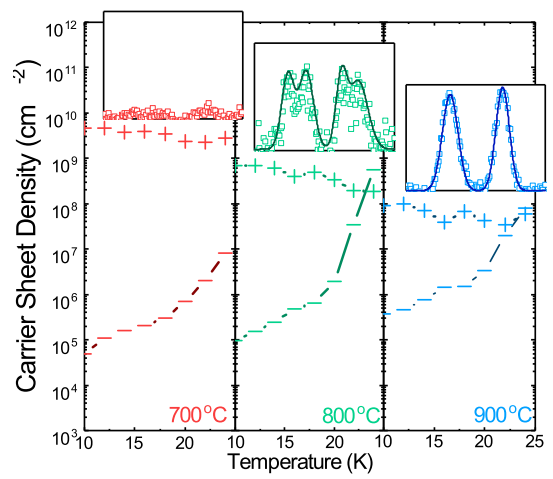

FIG. 10. 